\title{
Asymmetric Neighbor Discovery Protocol for Wireless Sensor Networks using Block Design
}

\author{
Woosik Lee*, Teuk-Seob Song**1 ${ }^{1}$, and Jong-Hoon Youn*** \\ * ICT Convergence and Integration Research Institute, Korea Institute of Civil \\ Engineering and Building Technology, South Korea \\ E-mail: leewoosik@kict.re.kr \\ **Division of Convergence Computer and Media, Mokwon University, South \\ Korea \\ E-mail:teukseob@mokwon.ac.kr \\ *** College of Information Science \& Technology, \\ University of Nebraska at Omaha, NE, USA \\ jyoun@unomaha.edu
}

\begin{abstract}
A number of neighbor discovery protocols (NDPs) based on block designs have been proposed for the application of sensor networks. However, most of these block designsbased NDPs in literature cannot support asymmetric duty cycles, whereas internet of things (IoT) applications prefer to support both symmetric and asymmetric duty operations. In order to address the lack of supporting asymmetric duty operations of block designs-based NDPs, we propose a new scheme called the Exclusive OR NDP, which constructs a new discovery schedule by combining two block designs with different duty cycles. The Exclusive OR NDP can support both symmetric and asymmetric duty cycles while preserving the performance of block designs-based NDPs. In this paper, we also present the results of our experimental study where we measure the effectiveness of the proposed approach by comparing the performance of the Exclusive OR NDP with other well-known NDPs.
\end{abstract}

Keywords: Internet of Things, Neighbor Discovery Protocol, Block Designs, Block Construction, Asymmetric Duty Cycles

\section{Introduction}

In the Internet of things (IoT), there are lots of heterogeneous sensing devices, which may have a different wakeup schedule and duty cycle. In a symmetric duty cycle network, all sensor nodes have the same duty cycles - i.e., they have the same ratio of the duration of the wakeup time to the period of one discovery cycle. On the other hand, if each node chooses its duty cycles independently, it is called an asymmetric duty cycle network. That is, a group of sensors may have a different ratio of the wakeup time within a period of a cycle.

Because a wide variety of monitoring devices can be networked together to collect and exchange data in IoT applications, finding their neighbors for communication is an indispensable part of IoT. A neighbor discovery protocol (NDP) is a representative scheme to find neighboring nodes. Because most of IoT applications desire to support both symmetric and asymmetric duty cycles, NPDs for IoT applications need to handle both symmetric and asymmetric duty cycles efficiently.

\footnotetext{
${ }^{1}$ Corresponding author: Teuk-Seob Song, Ph.D. Division of Convergence Computer and Media, Mokwon University, Daejeon, Korea 35349, E-mail: teukseob@mokwon.ac.kr
} 
In a symmetric duty cycle network, it was proved that a block designs-based approach is an optimal solution [5] to a neighbor discovery problem in terms of latency and energy consumption. However, the block-based NDP has one critical limitation in applying to neighbor discovery of IoT applications. In a typical IoT application, the duty cycle of wireless devices can be configured independently based on the application requirements and the amount of available power resources. Therefore, the lack of supporting neighbor discovery in asymmetric networks is a huge limitation of the block designs-based NDP. In this paper, we propose a new neighbor discovery scheme called the Exclusive OR-based NDP, which reduces the number of active slots while preserving the total length of the discovery cycle. Therefore, the number of active slots in the Exclusive OR-based NDP is smaller than that of the OR-based NDP, and the cycle length of the proposed approach is equal to that of the OR-based NDP.

This paper is organized as follows. Section 2 shows related works on NDPs. In Section 3 , we introduce a new asymmetric scheduling method using block designs. In Section 4, we illustrate our simulation environment and compare the performance of prominent NDPs with the proposed method. Finally, we conclude this paper in Section 5.

\section{Related Works}

Due to the increasing popularity of IoT applications in various fields, the importance and need of power saving neighbor discovery techniques have increased recently. In this section, we summarize previous research works focusing on the development of neighbor discovery scheduling for wireless sensor networks.

In [4], the authors proposed a scheduling method that uses $n \times n$ grid, called a quorum based neighbor discovery protocol. Since the method is simple, it can be applied to various systems easily. In Figure 1 (A), an example of the quorum based NDP with $n=16$ is shown. The sensor node $u_{1}$ wakes up at the slots listed in the first row and the second column of the $4 \times 4$ grid, and $u_{2}$ wakes up during the slots located in the third row and the third column of the grid. If the sensor $u_{1}$ and $u_{2}$ are synchronized, both of them wake up at the slots indexed 2 and 9. Thus, they can communicate with each other during these slots. Although the quorum-based approach guarantees the existence of common active slots between any pair of neighboring nodes, all sensors in the network must have the same duty cycle. Later, Zhang et al. proposed an optimal neighbor discovery protocol by using the combinatorial theory and block designs [8]. The authors also proved that the proposed block designs-based NDP is a theoretically optimal solution for neighbor discovery. Although the block designs based discovery scheduling method produces a theoretical optimal solution for neighbor discovery in a symmetric sensor network, it cannot be applied to asymmetric networks. So, in [3], an asymmetric neighbor discovery protocol for wireless sensor networks using two prime numbers was introduced, and a neighbor discovery protocol using only one prime number was proposed in [5]. In [5], the authors showed their method was more efficient than the method using a pair of prime numbers.

Recently, in [17], Choi et al, introduced a new neighbor discovery protocol called BAND based on the block design concept. Although BAND was a variation of the block designs-based scheme, it supports a more diverse set of duty cycles than the original method. Figure 1 (B) shows an example of BAND. To construct a new discovery schedule, it combines two binary incomplete block designs (BIBDs); one is called a base design and the other is called a replace design. In Figure 1 (B), the base design is a $(4,3$, $2)-\mathrm{BIBD}$, and the replace deign is a $(3,2,1)$-BIBD. Thus, the resulting schedule length is $4 \times 3=12$, and the number of active slots are $3 \times 2=6$. For any two sensors using an arbitrary row from the $12 \times 12$ grid shown in Figure 1 (B) for neighbor discovery, they have at least one common active slot due to the properties of BIBDs [17]. However, BAND [17] comes with the same weakness; it supports symmetric networks only. In [14], 
Lee $e t$ al, introduced a new approach called an OR-based neighbor discovery protocol that can support both symmetric and asymmetric neighbor discovery in sensor networks. In [15], they further extended the block design based method using a prime number in order to support a network with asymmetric duty cycles. However, the method extends the length of the discovery cycle significantly, and the discovery latency may increase considerably in the worst case.

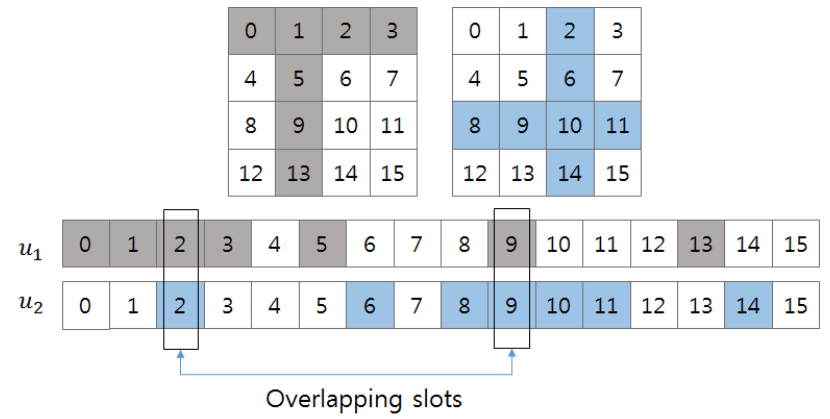

(A)

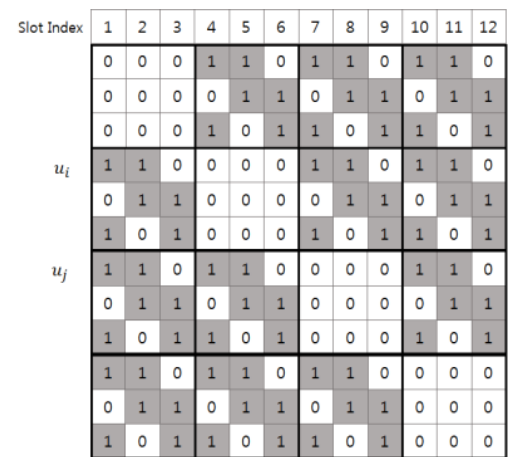

(B)

\section{Figure 1. (A) An Example of the Grid Quorum based NDP [4] and (B) the BIBD based BAND NDP [17].}

In this paper, we propose a new NDP for asymmetric networks using block designs. The duty cycle of the proposed approach is lower than that of the OR-based method. As the duty cycle is very tightly related to the power consumption of the network, the proposed method is more energy efficient than previous approaches in literature. Furthermore, our method does not increase the length of the discovery cycle, although it reduces the number of active slots. Therefore, our approach can support asymmetric neighbor discovery without compromising the worst case latency and energy efficiency.

\section{Asymmetric Scheduling Method using Block Design}

This section introduces the relationship between asymmetric neighbor discovery and BIBD-based block designs [9]. First of all, we introduce two definitions related to combinatorial block designs.

Definition 1. A design is a pair $(X, \mathcal{A})$ such that the following properties are satisfied:

$X$ is a set of elements called points, and

$A$ is a collection (i.e., multiset) of nonempty subsets of $X$ called blocks.

Balanced Incomplete Block Design (BIBD) is a well-studied type of designs that are applicable to various applications such as software testing, geometry, and cryptography [17].

Definition 2. Let $v, k$, and $\lambda$ be positive integers such that $v>k \geq 2$. A $(v, k, \lambda)$ Balanced Incomplete Block Design (which we abbreviate to $(v, k, \lambda)$-BIBD) is a design $(X$, A) such that the following properties are satisfied:

1) $|X|=v$,

2) Each block contains exactly $k$ points, and

3) Every pair of distinct points is contained in exactly $\lambda$ blocks.

For instance, let the set $X=\{1,2,3,4,5,6,7\}$ be given, and let the multiset $A$ $=\{\{1,2,4\},\{2,3,5\},\{3,4,6\},\{4,5,7\},\{1,5,6\},\{2,6,7\},\{1,3,7\}\}$ be given. Then, we can easily see that the design $(X, A)$ satisfies three properties listed in Definition 2 . Thus, $(X, A)$ is a $(7,3,1)$-BIBD. Note that a $(7,3,1)$-BIBD is not unique. For the given multiset 
$\mathscr{A}^{\prime}=\{\{1,2,3\},\{1,4,5\},\{1,6,7\},\{2,4,6\},\{2,5,7\},\{3,4,7\},\{3,5,6\}\}$, the $\left(X, \mathscr{A}^{\prime}\right)$ also satisfies the properties 1$), 2)$ and 3$)$ in Definition 2 . Thus, $\left(X, A^{\prime}\right)$ is also a $(7,3,1)$-BIBD.

According to the results of the study on block designs, for given positive numbers $v, k$, and $\lambda$, the existence of $(v, k, \lambda)$-BIBDs is not known. However, if $h$ is a power of a prime, the existence of $\left(h^{2}+h+1, h+1,1\right)$-BIBD is proved [9]. It is a special case of $\lambda=1$ in the $(v, k, \lambda)$-BIBD. Due to the guaranteed availability of $\left(h^{2}+h+1, h+1,1\right)$-BIBDs, in this study, we focus on the design of neighbor discovery schedules based on $\left(h^{2}+h+\right.$ $1, h+1,1)$-BIBDs.

We assume a wireless sensor toggles the active and sleep modes during the neighbor discovery process. These modes can be denoted by a binary number, where " 1 " represents the active mode, and " 0 " represents the sleep mode.

Definition 3. Suppose that a wireless sensor node $u$ has a discovery schedule with a cycle $T$, we denote $u$ 's discovery schedule as a sequence $S_{u}^{T}=<a_{1}, a_{2}, a_{3}, \ldots, a_{T-1}, a_{T}>$ such that for all $i$ in $[1, T]$ and $a_{i}=0$ or 1 . In $S_{u}^{T}$, the $i^{\text {th }}$ binary number represents the $i^{\text {th }}$ discovery slot in the cycle $T$. When $a_{i}=1$, the $i^{\text {th }}$ discovery slot is called as an active slot where the sensor $u$ must stay awake, whereas the sensor turns off its radio during the $i^{\text {th }}$ slot, called a sleep slot, if $a_{i}=0$.

Note that a sensor needs to repeat the discovery schedule a number of times during the discovery phase. This is because a pair of neighboring sensors may not be able to discover each other during their overlapped active slots due to a message collision, channel interference or impulsive noise. Therefore, the sequence $S_{u}^{T}$ satisfies the equation $a_{i+n T}=$ $a_{i}$ for all $i$ in $[1, T]$ and $n \in Z^{+}$.

If a sensor $u$ uses a BIBD block $A_{u}$ for neighbor discovery where $A_{u}$ is a block from $\mathrm{a}(v, k, \lambda)$-BIBD, then its discovery schedule is given by $S_{u}^{v}=\left\langle a_{1}, a_{2}, a_{3}, \ldots, a_{v-1}, a_{v}\right\rangle$ such that, for $1 \leq i \leq v, a_{i}=1$ if $i \in A_{u}$, otherwise, $a_{i}=0$. Since the cycle length of the sensor $u$ is the cardinality of the set $X$, i.e. $|X|=v$, the sequence $S_{u}^{v}$ satisfies the equation $a_{i+n v}=a_{i}$ for all $i$ in $[1, v]$ and $n \in Z^{+}$. The following figure depicts the first fourteen slots of the discovery schedule based on the $\{1,2,4\}$ block in the $(7,3,1)$-BIBD. In Figure 2, the black box represents an active slot, and the white box represents a sleep slot. Since the schedule has a cycle of length 7, the indexes of the active slots are 1, 2, 4, $1+7,2+7,4+7,1+2 \times 7,2+2 \times 7,4+2 \times 7$, and so on.

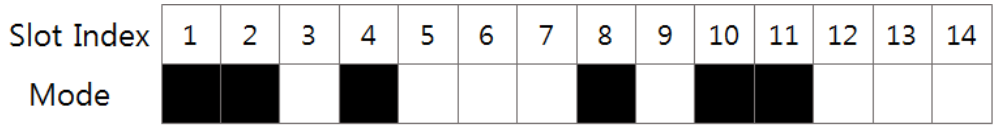

\section{Figure 2. An Example of a Schedule based on the $\{1,2,4\}$ Block of the $(7,3,1)$-BIBD}

Next, we define the common active slots between two discovery schedules $S_{u_{1}}^{v_{1}}$ and $S_{u_{2}}^{v_{2}}$. Since the cycle lengths of $S_{u_{1}}^{v_{1}}$ and $S_{u_{2}}^{v_{2}}$ are $v_{1}$ and $v_{2}$, respectively, we will consider the common active slots within the LCM (least common multiple) of $v_{1}$ and $v_{2}$.

Definition 4. The operation $\wedge$ between two discovery schedules $S_{u_{1}}^{v_{1}}$ and $S_{u_{2}}^{v_{2}}$ represents the set indexes of common active slots between two schedules. $S_{u_{1}}^{v_{1}} \wedge S_{u_{2}}^{v_{2}}=\left\{i \mid\right.$ for $1 \leq i \leq L, a_{i}=b_{i}=1$, where $a_{i}$ in $S_{u_{1}}^{v_{1}}$ and $b_{i}$ in $\left.S_{u_{2}}^{v_{2}}\right\}$.

Also, the notation $|X|$ means the cardinality of a set $X$. Thus, $\left|S_{u_{1}}^{v_{1}} \wedge S_{u_{2}}^{v_{2}}\right|$ denotes the number of common active slots between $S_{u_{1}}^{v_{1}}$ and $S_{u_{2}}^{v_{2}}$ within a cycle.

The following lemma shows that the proposed BIBD-based discovery schedule guarantees the existence of at least one common active slot within a length of a cycle for any pair of randomly selected two sensors. 
Lemma 1. Let $(X, \mathcal{A})$ be a symmetric $(v, k, \lambda)$-BIBD. Let $A_{i}$ and $A_{j}$ be two different blocks in $\mathcal{A}$. Let $S_{u_{i}}^{v}$ and $S_{u_{j}}^{v}$ be two discovery schedules derived from the block $A_{i}$ and $A_{j}$, respectively. Then the schedule $S_{u_{i}}^{v}$ and $S_{u_{j}}^{v}$ have $\lambda$ common active slots; $\left|S_{u_{i}}^{v} \wedge S_{u_{j}}^{v}\right|=\lambda$.

Proof. Since the two $A_{i}$ and $A_{j}$ blocks in the symmetric $(v, k, \lambda)$-BIBD, by the theorem 1.1.4. in [9], $A_{i}$ and $A_{j}$ have $\lambda$ common elements. It means that the schedules $S_{u_{i}}^{v}$ and $S_{u_{j}}^{v}$ have $\lambda$ common active slots. Therefore, we can get $\left|S_{u_{i}}^{v} \wedge S_{u_{j}}^{v}\right|=$ $\lambda$.

The BIBD-based NDP protocol is proved to be an optimal solution for neighbor discovery in wireless sensor networks with a symmetric duty cycle [5]. However, BIBDbased NDPs have a limitation in applying the method for asymmetric WSNs. As shown in Figure 3 , two sensor nodes, $u_{1}$ and $u_{2}$, have no common active slot. The sensor node $u_{1}$ uses the $\{1,2,4\}$ block in the $(7,3,1)$-BIBD, i.e. $S_{u_{1}}^{7}=\{1,1,0,1,0,0,0\}$, and $u_{2}$ is based on the $\quad\{3,6,7,12,14\}$ block in the $(21,5,1)-B I B D$, i.e. $S_{u_{2}}^{21}=\{0,0,1,0,0,1,1,0,0,0,0,1,0,1,0,0,0,0,0,0,0\}$. Since the 7 is a factor of 21 , this pattern repeats infinitely. Therefore, there is no chance that two neighboring sensors $u_{1}$ and $u_{2}$ discover each other since there is no common active slot between $S_{u_{1}}^{7}$ and $S_{u_{2}}^{21}$.

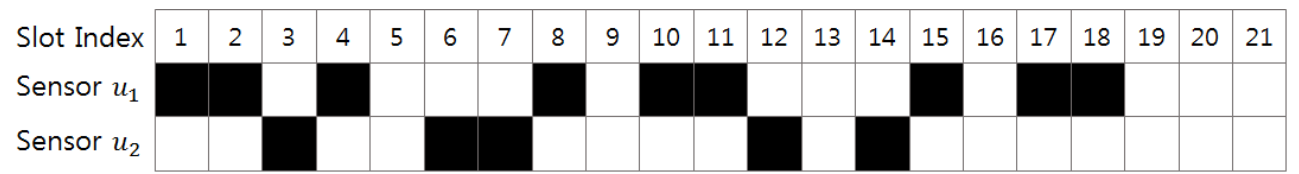

Figure 3. An Example of Two Asymmetric BIBD-based Schedules without any Common Active Slot

The proposed Exclusive OR-based NDP combines two different blocks selected from $(v, k, \lambda)$-BIBD designs where $v$ is the length of the block, $k$ is the number of active slots in each block, and $\lambda$ is the number of common active slots between a pair of blocks. In [14, $15]$, it was shown that $(v, k, \lambda)$-BIBD cannot support asymmetric duty cycle. In order to solve this problem, we propose the new NDP method that combines several $(v, k, \lambda)$ BIBD blocks using a bitwise exclusive OR (XOR) operation.

Definition 5. Let $A$ and $B$ be blocks selected from $\left(v_{1}, k_{1}, \lambda_{1}\right)$-BIBD and $\left(v_{2}, k_{2}, \lambda_{2}\right)$ BIBD, respectively. Assume $S_{u_{1}}^{v_{1}}$ and $S_{u_{2}}^{v_{2}}$ are the discovery schedules generated from the blocks $A$ and $B$, respectively. The Exclusive OR-based schedule $S_{u_{1}}^{v_{1}} \oplus S_{u_{2}}^{v_{2}}$ is defined by a sequence $S_{u_{1}}^{v_{1}} \oplus S_{u_{2}}^{v_{2}}=<a_{1} \oplus b_{1}, a_{2} \oplus b_{2}, a_{3} \oplus b_{3}, \ldots, a_{L-1} \oplus b_{L-1}, a_{L} \oplus b_{L}>$, where for $1 \leq i \leq L, a_{i}$ in $S_{u_{1}}^{v_{1}}$ and $b_{i}$ in $S_{u_{2}}^{v_{2}}$, the notation $\bigoplus$ is the logical XOR operation, and $L$ is the least common multiple (LCM) of $v_{1}$ and $v_{2}$.

Figure 4 shows the Exclusive OR-based block combination of $(7,3,1)$-BIBD and $(3,2,1)$ BIBD.

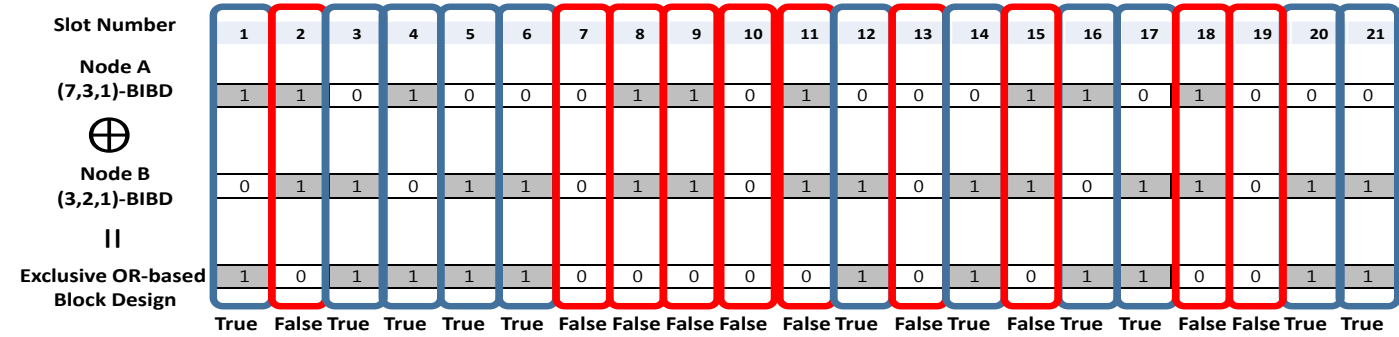

Figure 4. An Example of the Exclusive OR-based Block Combination 
If two sensor nodes use the Exclusive OR-based NDP generated using the $\oplus$ operation shown in Definition 5, they must have common active slots. Next Theorem guarantees the existence of common active slots when sensor nodes use the proposed exclusive ORbased NDP.

Theorem 1. Let $A$ and $B$ be blocks from two different $\left(v_{1}, k_{1}, \lambda_{1}\right)$-BIBD and $\left(v_{2}, k_{2}, \lambda_{2}\right)$-BIBD, respectively. If $S_{u_{1}}^{v_{1}}$ and $S_{u_{2}}^{v_{2}}$ are the schedules generated from the blocks $A$ and $B$, respectively, then $\left|S_{u_{1}}^{v_{1}} \wedge\left(S_{u_{1}}^{v_{1}} \oplus S_{u_{2}}^{v_{2}}\right)\right| \geq 1$.

Proof. Since $v_{1}$ and $v_{2}$ are different integers, without loss of generosity, we can assume that $v_{2}=\max \left\{v_{1}, v_{2}\right\}$. Since $v_{2}$ is greater than $v_{1}, k_{2}$ is greater than $k_{1}$. It denotes that the number of active slots in $u_{1}$ is greater than the number of active slots of in $u_{2}$ within $L$, where the $L=L C M\left(v_{1}, v_{2}\right)$. Thus, there are some $i$ in $1 \leq i \leq L$ such that $a_{i}=1$, where $a_{i} \in S_{u_{1}}^{v_{1}}$, and $b_{i}=0$, where $b_{i} \in S_{u_{2}}^{v_{2}}$. Therefore, $i \in S_{u_{1}}^{v_{1}} \wedge\left(S_{u_{1}}^{v_{1}} \oplus S_{u_{2}}^{v_{2}}\right)$. As a result, we know that $\left|S_{u_{1}}^{v_{1}} \wedge\left(S_{u_{1}}^{v_{1}} \oplus S_{u_{2}}^{v_{2}}\right)\right| \geq 1$. It means that any discovery schedule derived from the $\left(v_{1}, k_{1}, \lambda_{1}\right)$-BIBD $\left(S_{u_{1}}^{v_{1}}\right)$ and any schedule generated by combining two schedules $S_{u_{1}}^{v_{1}}$ and $S_{u_{2}}^{v_{2}}\left(S_{u_{1}}^{v_{1}} \oplus S_{u_{2}}^{v_{2}}\right)$ have at least one common active slot within the period $L$.

\section{Simulation Study}

In order to compare the performance of our proposed NDP with other previously known NDPs, we have developed a simulation environment using TOSSIM [20]. The following protocols were implemented for the performance comparison; the OR-based NDP [14], U-Connect [5], Disco [3], and Todis [19]. For the configurations of the simulation environment, we assume 50 sensors are randomly spread over a $100 \mathrm{~m} \times$ $100 \mathrm{~m}$ sending field. For communication between sensors, the CC2420 radio modules with $2.4 \mathrm{GHz}$ [21] were adapted for this study, and the log-distance path loss model [22] was used for the radio propagation model. We assume the length of each discovery slot is 15 milliseconds, and a sensor node wakes up and exchanges discovery messages asynchronously at the beginning of its discovery slot. The simulation configuration parameters are summarized in Table 1.

Table 1. Configurations of the Simulation Environment

\begin{tabular}{|c|c|}
\hline Properties & Values \\
\hline Network Topology & Random topology \\
\hline Experimental Area & $(100 \mathrm{~m} \times 100 \mathrm{~m})$ field \\
\hline Radio Module & CC1000 \\
\hline The number of sensors & 50 \\
\hline Event Trigger Time & $15 \mathrm{~ms}$ \\
\hline Energy Calculator Module & PowerTOSSIM Module \\
\hline Link Layer Model & USC Link Model \\
\hline Channel Access Scheme & CSMA/CA \\
\hline Radio Propagation Model & Log-Distance Path Loss Model \\
\hline Neighbor Discovery Protocols & $\begin{array}{c}\text { OR, U-Connect, Exclusive OR, Disco, } \\
\text { Todis }\end{array}$ \\
\hline
\end{tabular}

To consider an asymmetric sensor network where each sensor picks its duty cycle independently, we define a variable $\mathrm{R}$ as a ratio of the high duty cycle over the low duty cycle in the networks [5]. For example, if there are two groups of sensors operating at $10 \%$ duty cycle and $5 \%$ duty cycles, the ratio $\mathrm{R}$ is $10 \% / 5 \%=2$. The simulation scenarios 
include four different $\mathrm{R}$ values varying from 1 to $10 ; \mathrm{R}=1$ (all $10 \%$ duty cycles), $\mathrm{R}=2$ (10\% vs. $5 \%$ duty cycles), $\mathrm{R}=5$ ( $10 \%$ vs. $2 \%$ duty cycles), and $\mathrm{R}=10$ ( $10 \%$ vs. $1 \%$ duty cycles). In this study, we consider two performance metrics; latency and energy consumption. Latency of a sensor is the amount of time taken until the last neighbor sensor is discovered. Energy consumption is the amount of energy consumed during the entire neighbor discovery phase.

Table 2. Parameters of the Discovery Schedule

\begin{tabular}{|c|c|c|c|c|}
\hline Protocol & $\mathrm{R}=1$ & $\mathrm{R}=2$ & $\mathrm{R}=5$ & $\mathrm{R}=10$ \\
\hline Exclusive & $(91,10)$ & $(91,10)$ & $(91,10)$ & $(91,10)$ \\
OR & $(91,10)$ & $(381,56)$ & $(2451,310)$ & $(9507,1125)$ \\
\hline \multirow{2}{*}{ OR-based } & $(91,10)$ & $(91,10)$ & $(91,10)$ & $(91,10)$ \\
& $(91,10)$ & $(381,37)$ & $(2451,67)$ & $(9507,117)$ \\
\hline \multirow{2}{*}{ Disco } & $(17,23)$ & $(17,23)$ & $(17,23)$ & $(17,23)$ \\
& $(17,23)$ & $(37,43)$ & $(93,103)$ & $(197,199)$ \\
\hline \multirow{2}{*}{ U-Connect } & $(13,13)$ & $(13,29)$ & $(13,73)$ & $(13,149)$ \\
\hline \multirow{2}{*}{ Todis } & $n=29$ & $n=29$ & $n=29$ & $n=29$ \\
& $n=29$ & $n=59$ & $n=149$ & $n=299$ \\
\hline
\end{tabular}

Table 2 shows the parameters of the discovery schedules for the NDPs included in the study. For the Exclusive-OR and the OR-based NDPs, the two values within the parentheses in the table denote the total schedule length $v$ and the number of active slots $k$. For Disco and U-Connect, the values within the parentheses represent two prime numbers selected for the discovery schedules. For Toids is triple odd number based NDP, the $n$ means the middle integer among the three odd integers.

Through the simulation study, we compare the performance of various NDPs in terms of the maximum and average latencies. According to the results shown in Figure 6, Block-based NDPs clearly outperform the Prime-based NDPs such as Disco, U-Connect, and Todis. In particular, a Block-based NDP stably finds neighbors within a total length of one cycle, but Prime-based NDPs occasionally experience extremely long discovery latency. The experimental results shown in Figure 6 confirm that the Exclusive OR-based NDP outperforms other NDPs in terms of latency, and the performance gab between the proposed approach and other NDPs increases as the $\mathrm{R}$ value increases. In particular, when $\mathrm{R}$ is 10, the worst-case latency of Disco is $688.5575 \mathrm{~s}$ and that of the Exclusive OR-based NDP is 137.2932 s. Thus, the worst-case discovery latency of the proposed NDP is only $20 \%$ of the maximum latency of Disco. When $\mathrm{R}=10$, the measured average discovery latency of the Exclusive OR-based and Disco are 23.07s and 69.08s respectively; note that the average latency of Disco is about three times longer than that of the proposed method. 

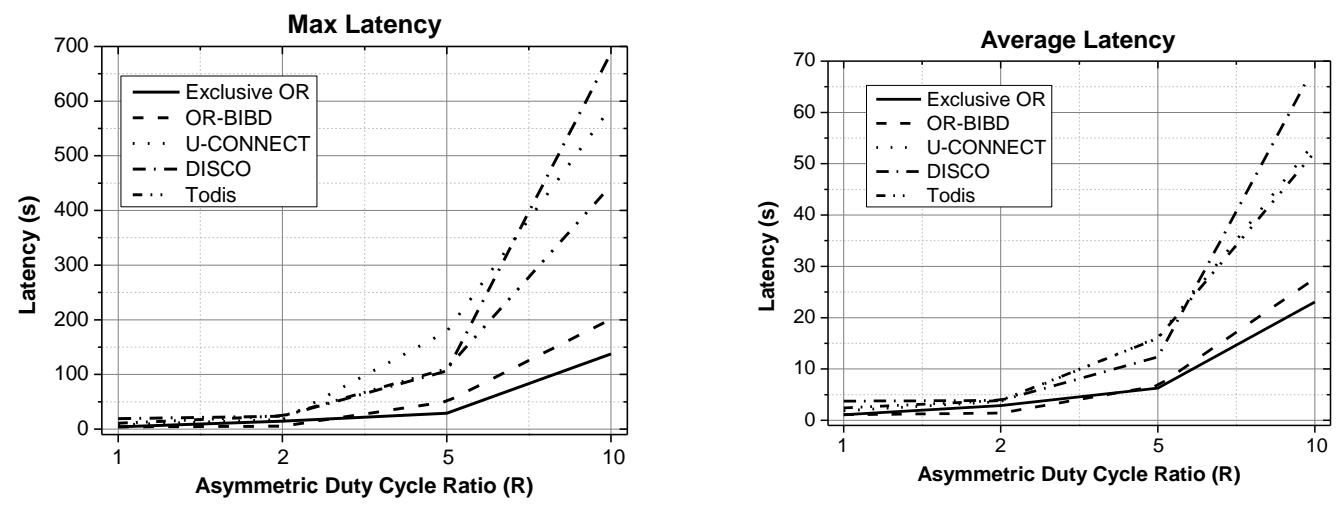

\section{Figure 6. Average and Max Latency each Duty Cycle in Asymmetric Scenarios}

The trends of the measured energy consumption are similar to the measured latency variations. This is because the energy consumption is the total energy use of a sensor until the sensor finds all neighbors. For example, if one sensor does not find all neighboring nodes, it continually sends a discovery message to their neighbors. These periodic transmissions of discovery messages for the entire duration of the discovery phase may result in a significant amount of energy consumption. Similarly, for an NDP with a short latency, each sensor spends a relatively small quantity of energy. The number of active slots within a cycle is an important factor of defining the energy consumption of sensors. If all NDPs have the same duty cycle, then NDPs with the shortest total length will be better than other NDPs in terms of latency and energy consumption.

The simulation results shown in Figure 7 demonstrate that the Exclusive OR-based NDP can significantly reduce energy consumption during the neighbor discovery phase, and prolong the lifetime of sensors by reducing the energy consumption in neighbor discovery. When $\mathrm{R}$ is 10 , the energy consumption of the Exclusive OR-based NDP is up to $35 \%$ less than that of the OR-based NDP. Note that the maximum energy consumptions of the OR-based and the Exclusive OR-based NDPs are $995.0386 \mathrm{~mJ}$ and $639.0833 \mathrm{~mJ}$, respectively.
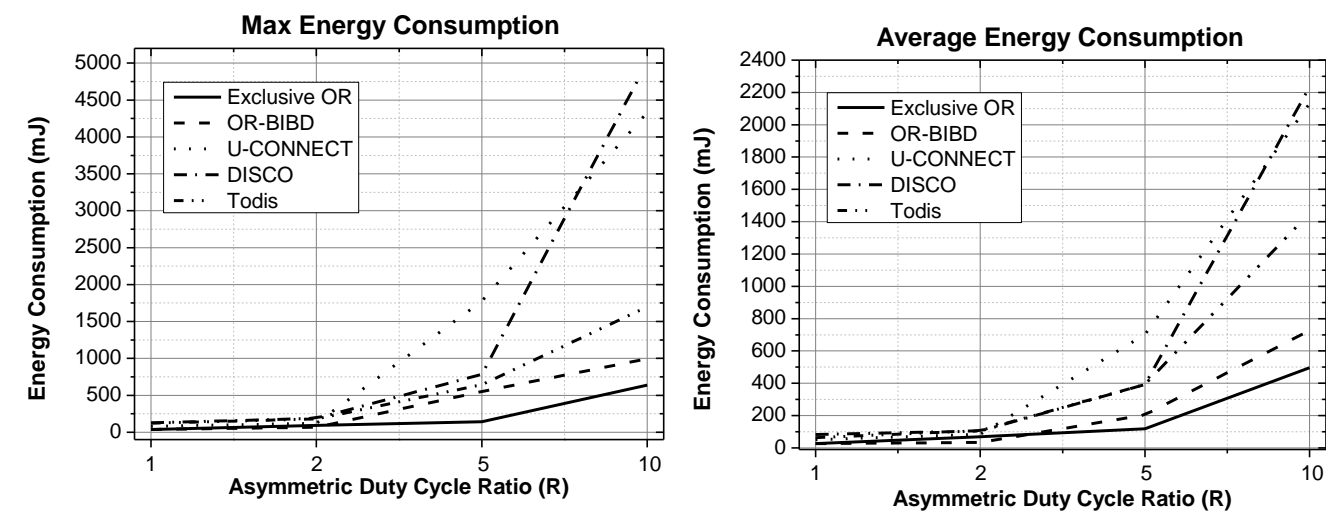

\section{Figure 7. Max and Average Energy Consumption each Duty Cycle in Asymmetric Scenarios.}

Based on the experimental results shown above, we conclude that the Exclusive ORbased NDP is clearly outperforming other NDPs included in the study. 


\section{Conclusion}

In the applications of IoT, sensor nodes need to use a good neighbor discoveryscheduling scheme because they frequently have to find their neighbors to exchange packets. In particular, IoT sensor nodes may use a tiny battery which has a limit power resource, and the physical network topology may change rapidly and unpredictably due to node failures, battery exhaustion, new deployment, or moving out of range. Therefore, in a typical IoT application, each node needs to update its neighbor list periodically, and the design and development of energy-efficient neighbor discovery protocols is a critical building block of a successful implementation of IoT applications.

In this paper, we proposed a new combinatorial block design based NDP called an Exclusive OR-based NDP, in order to address asymmetric neighbor discovery problem in sensor networks. The proposed method reduces the number of active slots of the ORbased NDP without extending the length of the discovery cycle. The experimental results shown in Section 4 clearly demonstrate that the superiority of the proposed approach over other NDPs in the literatures such the OR-based NDP, U-Connect, Disco, and Todis.

\section{Acknowledgments}

This research was supported by Basic Science Research Program through the National Research Foundation of Korea (NRF) funded by the Ministry of Education, Science and Technology (NRF-2015R1D1A1A01058786).

\section{References}

[1] K. Han, J. Luo, Y. Liu and A.V. Vasilakos, "Algorithm Design for Data Communications in DutyCycled Wireless Sensor Networks: A Survey”, IEEE Communications Magazine, vol. 51, no. 7, (2013), pp.107-113.

[2] W. Sun, Z. Yang, X. Zhang and Y. Liu, "Energy-Efficient Neighbor Discovery in Mobile Ad Hoc and Wireless Sensor Networks: A Survey”, IEEE Communications Surveys \& Tutorials, vol.16, no. 3 (2014), pp.1448-1459.

[3] P. Dutta and D. Culler, "Practical Asynchronous Neighbor Discovery and Rendezvous for Mobile Sensing Applications", Proceedings of the 6th ACM Conference on Embedded Network Sensor Systems, (2008), pp.71-84.

[4] J. R. Jiang, Y.C. Tseng, C.S. Hsu and T.H. Lai, "Quorum-based Asynchronous Power-saving Protocols for IEEE 802.11 Ad Hoc Networks", Mobile Networks and Applications, vol. 10, no.1 (2005), pp.169181.

[5] A. Kandhalu, K. Lakshmanan, and R. Rajkumar, "U-connect: A Low-Latency Energy-efficient Asynchronous Neighbor Discovery Protocol," Proceedings of the 9th ACM/IEEE International Conference on Information Processing in Sensor Networks, (2010), pp.350-361.

[6] M.J. McGlynn and S.A. Borbash, "Birthday Protocols for Low Energy Deployment and Flexible Neighbor Discovery in Ad Hoc Wireless Networks", Proceedings of the 2nd ACM International Symposium on Mobile Ad Hoc Networking \& Computing, (2001), pp.137-145.

[7] Y.C. Tseng, C.S. Hsu and T.Y. Hsieh, "Power-Saving Protocols for IEEE 802.11-based Multi-Hop Ad Hoc Networks", Proceedings of the 21Annual Joint Conference of the IEEE Computer and Communications Societies, (2002), pp.200-209.

[8] R. Zheng, J.C. Hou and L. Sha, "Optimal Block Design for Asynchronous Wake-Up Schedules and Its Applications in Multihop Wireless Networks", IEEE Transactions on Mobile Computing, (2006), pp.1228-1241

[9] I. Andreson, "Combinatorial designs and Tournaments", Clarendon Press Oxford Science Publication, (1997).

[10] P. Levis, N. Lee, M. Welsh and D. Culler, "TOSSIM: Accurate and Scalable Simulation of Entire TinyOS Applications", Proceedings of the 1st International Conference on Embedded Networked Sensor Systems, (2003), pp.126-137.

[11] E. Perla, A.O. Cathain, R.S. Carbajo, M. Huggard and C.M. Goldrick, "PowerTOSSIM Z: Realistic Energy Modelling for Wireless Sensor Network Environments", Proceedings of the 3nd ACM Workshop on Performance Monitoring and Measurement of Heterogeneous Wireless and Wired Networks, (2008), pp.35-42.

[12] CC2420, http://inst.eecs.berkeley.edu/ cs150/Documents/CC2420.pdf.

[13] USC Topology, http://www.tinyos.net/tinyos-2.x/doc/html/tutorial/usc-topologies.html. 
[14] W.S. Lee, S.G. Choi, T.S. Song, N.G. Kim and J.H. Youn, "OR-based Block Combination for Asynchronous Asymmetric Neighbor Discovery Protocol", International Journal of Control and Automation, vol. 8, no. 3, (2015), pp.45-52.

[15] W.S. Lee, I.H. Youn, T.S. Song, N.G. Kim and J.H. Youn, "Prime Block Design for Asynchronous Wake-Up Schedules in Wireless Sensor Networks", IEEE Communications Letters, vol. 20, no. 7, (2016), 1437-1440.

[16] T.W. Song, H.H. Park and S.H. Pack, "Probabilistic Neighbor Discovery Algorithm in Wireless Ad Hoc Networks", Journal of Information and Communication Convergence Engineering, vol. 39B, no. 9, (2014), pp.561-569.

[17] S. Choi, W. Lee, T.S. Song and J.H. Youn, "Block Design-Based Asynchronous Neighbor Discovery Protocol for Wireless Sensor Networks", Journal of Sensors, vol. 2015, Art. No. 951652, (2015), pp. 111.

[18] M. Bakht, M. Trower and R. Kravets, "Searchlight: Won't You be My Neighbor?", Proceedings of ACM MobiCom, (2012), pp.185-196.

[19] L. Chen, R. Fan, K. Bian, L. Chen, M. Gerla, T. Wang and X. Li, "On Heterogeneous Neighbor Discovery in Wireless Sensor Networks", Proceedings of IEEE INFOCOM, (2015), pp.693-701.

[20] P. Levis, N. Lee, M. Welsh and D. Culler, "TOSSIM: Accurate and Scalable Simulation of Entire TinyOs Applications," Proceedings of ACM SenSys, (2003), pp. 126-137.

[21] CC2420, http://inst.eecs.berkeley.edu/ cs150/Documents/CC2420.pdf.

[22] USC Topology, http://www.tinyos.net/tinyos-2.x/doc/html/tutorial/usc-topologies.html.

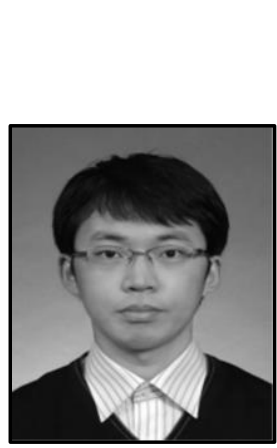

\section{Authors}

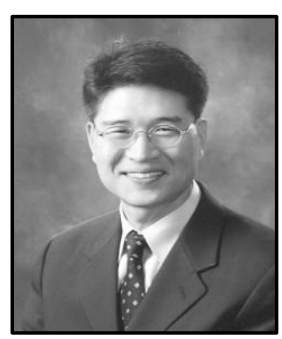

Woosik Lee, he received his B.S., M.S., and $\mathrm{PhD}$ degrees in Computer Science from Kyonggi University, Korea in 2009, 2011, and 2016, respectively. He is currently a researcher of ICT Convergence and Integration Research Institute in Korea institute of civil engineering and building technology. His research interests include wireless systems, sensor networks, smart city and energy management protocols.

Teuk-Seob Song, he received his Ph.D. degree in Computer Science in 2006 and Ph.D. degree in Mathematics from Yonsei University in 2001, respectively. He is currently an associate professor in the Division of Convergence Computer and Media at Mokwon University in Korea. His research interests include 3D virtual environment, annotation technology, and wireless sensor networks. He is a member of the Institute of Electrical and Electronics Engineers (IEEE), Korea Information Science Society, the Korea Information Processing Society, and the Korea Multimedia Society.

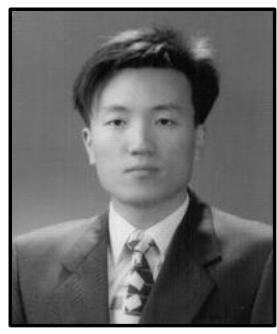

Jong-Hoon Youn, he received his M.S. and Ph.D. degrees in Computer Science from Oregon State University in 1999 and 2002 , respectively. Since 2002, he has been a faculty of the University of Nebraska at Omaha. His research interests include wireless communication and mobile computing, sensor networks, and mobility monitoring systems. 